\title{
Strict u-ideals in Banach spaces
}

\author{
by \\ Vegard Lima (Ålesund) and Åsvald Lima (Kristiansand)
}

\begin{abstract}
We study strict u-ideals in Banach spaces. A Banach space $X$ is a strict $\mathrm{u}$-ideal in its bidual when the canonical decomposition $X^{* * *}=X^{*} \oplus X^{\perp}$ is unconditional. We characterize Banach spaces which are strict $u$-ideals in their bidual and show that if $X$ is a strict $\mathrm{u}$-ideal in a Banach space $Y$ then $X$ contains $c_{0}$. We also show that $\ell_{\infty}$ is not a u-ideal.
\end{abstract}

1. Introduction. Strict u-ideals were introduced by Godefroy, Kalton and Saphar in [9]. Let $X$ be a subspace of a Banach space $Y$. We will say that $X$ is a summand of $Y$ if it is the range of a contractive projection and that $X$ is an ideal in $Y$ if $X^{\perp}$ is the kernel of a contractive projection on $Y^{*}$.

A norm one operator $\phi: X^{*} \rightarrow Y^{*}$ such that $\phi\left(x^{*}\right)(x)=x^{*}(x)$ is said to be a Hahn-Banach extension operator. The set of all such $\phi$ is denoted by $\mathrm{HB}(X, Y)$. For every $\phi \in \mathrm{B}(X, Y)$ we have

$$
Y^{*}=X^{\perp} \oplus \phi\left(X^{*}\right) .
$$

Let $i_{X}$ be the natural embedding $i_{X}: X \rightarrow Y$. Then $P_{\phi}=\phi \circ i_{X}^{*}$ is a norm one projection on $Y^{*}$ with $\operatorname{ker} P_{\phi}=X^{\perp} . X$ is an ideal in $Y$ if and only if $\mathrm{B}(X, Y) \neq \emptyset$ (see [8, Theorem 2.4]). If we have $\left\|x^{\perp}+\phi\left(x^{*}\right)\right\|=\left\|x^{\perp}-\phi\left(x^{*}\right)\right\|$ for all $x^{\perp} \in X^{\perp}$ and $x^{*} \in X^{*}$ we say that $X$ is a u-ideal in $Y$ and that $\phi$ is unconditional. Note that $\phi$ is unconditional if and only if $\left\|I-2 P_{\phi}\right\|=1$. We get the well-known notion of an $M$-ideal ([3], [12]) if $\left\|x^{\perp}+\phi\left(x^{*}\right)\right\|=$ $\left\|x^{\perp}\right\|+\left\|\phi\left(x^{*}\right)\right\|$ for all $x^{\perp} \in X^{\perp}$ and $x^{*} \in X^{*}$.

We get another useful viewpoint by defining a norm one operator $T_{\phi}$ : $Y \rightarrow X^{* *}$ by

$$
\left\langle i_{X}^{*} y^{*}, T_{\phi}(y)\right\rangle=\left\langle y, P_{\phi}\left(y^{*}\right)\right\rangle
$$

for all $y \in Y$ and $y^{*} \in Y^{*}$. Then $T_{\phi}(x)=x$ for all $x \in X$. Note that $T_{\phi}=\left.\phi^{*}\right|_{Y}$.

2010 Mathematics Subject Classification: Primary 46B04, 46B20.

Key words and phrases: strict u-ideal, u-summand. 
$X$ is a strict ideal in $Y$ if there is a $\phi \in \mathrm{B}(X, Y)$ such that $\phi\left(X^{*}\right)$ is norming. In this case $\phi$ is called strict. That $\phi$ is strict is equivalent to the existence for every $y \in Y$ and $\varepsilon>0$ of an $x^{*} \in B_{X^{*}}$ such that

$$
\|y\|-\varepsilon<\left\langle\phi\left(x^{*}\right), y\right\rangle=\left\langle x^{*}, T_{\phi}(y)\right\rangle .
$$

Since $\left|\left\langle x^{*}, T_{\phi}(y)\right\rangle\right| \leq\left\|T_{\phi}\right\|\left\|x^{*}\right\|\|y\|$ we see that $\phi$ is strict if and only if $T_{\phi}: Y \rightarrow X^{* *}$ is isometric.

In this paper we study strict u-ideals, i.e. ideals for which the HahnBanach extension operator is both strict and unconditional. Godefroy, Kalton and Saphar note in the introduction to their paper [9] that the theory of $\mathrm{u}$-ideals is much less satisfactory and complete than in the complex case of h-ideals (which we will not discuss). We aim to fill a few of the gaps in the theory of u-ideals.

In Section 2 we use the local and geometric description of u-ideals the authors obtained in [15] to develop similar tools needed to study strict uideals. We obtain a characterization of when a space of codimension one is a strict $\mathrm{u}$-ideal (see Theorem 2.4), and when a Banach space is a strict u-ideal in its bidual (see Theorems 2.8 and 2.9). Some of these results were first shown by Godefroy, Kalton and Saphar under the assumption that $X$ was separable or did not contain $\ell_{1}$. We also show that if $X$ is a non-trivial subspace of a Banach space $Y$, then $X$ contains a copy of $c_{0}$ whenever $X$ is a strict u-ideal in $Y$. In Theorem 2.12 we show that if a dual space $X^{*}$ is a u-ideal in its bidual, then it is in fact a u-summand. In particular, it can never be a strict $\mathrm{u}$-ideal. The proof relies on the fact that $\ell_{\infty}$ is not a $\mathrm{u}$-ideal in its bidual (see Theorem 2.11).

In Section 3 we look at denting points and strongly exposed points in the unit ball of the dual of $X$ when $X$ is a strict u-ideal in its bidual.

We use standard Banach space notation. For a Banach space $X, B_{X}$ is the closed unit ball and $S_{X}$ is the unit sphere. The canonical embedding $X \rightarrow X^{* *}$ is denoted by $k_{X}$. If $A$ is a subset of $X, \operatorname{span}(A)$ is the linear span of $A$ and $\operatorname{conv}(A)$ is the convex hull of $A$.

We consider real Banach spaces only.

2. Strict u-ideals. First we show that to check whether a $\mathrm{u}$-ideal is strict or not it is enough to check one direction at a time.

Proposition 2.1. Assume $X$ is a u-ideal in $Y$. Then $X$ is a strict u-ideal in $Y$ if and only if $X$ is a strict $u$-ideal in $\operatorname{span}(X,\{y\})$ for all $y \in Y$.

Proof. Let $\phi \in \mathrm{B}(X, Y)$ be unconditional. As noted in the introduction, $\phi$ is strict if and only if $T_{\phi}$ is isometric (notation of (1.1)). But by Lemma 2.2 and 3.1 in [15], $T_{\phi}(y)$ is uniquely and locally determined. 
Recall that an element $c$ in a convex set $K$ is a center of symmetry if $2 c-x \in K$ for all $x \in K$.

Proposition 2.2. If $X$ is a strict u-ideal in $Y$ then every element of $\mathrm{HB}(X, Y)$ is strict.

Proof. Assume $\phi \in \mathrm{B}(X, Y)$ is unconditional and strict. Then $\phi$ is a center of symmetry in $\mathrm{B}(X, Y)$ (see e.g. [1, Proposition 2.2]) so that $2 \phi-\psi \in$ $\mathrm{BB}(X, Y)$ for all $\psi \in \mathrm{B}(X, Y)$. Let $\psi \in \mathrm{B}(X, Y)$ and $y \in Y$. Then

$$
\|y\| \geq\left\|\left(2 T_{\phi}-T_{\psi}\right)(y)\right\| \geq 2\left\|T_{\phi}(y)\right\|-\left\|T_{\psi}(y)\right\|=2\|y\|-\left\|T_{\psi}(y)\right\| .
$$

Hence $\left\|T_{\psi}(y)\right\|=\|y\|$ and $\psi$ is strict.

Let us introduce some more notation. Assume $X$ is a closed subspace of a Banach space $Y$. For each $y \in Y \backslash X$ define

$$
D_{y}=X^{* *} \cap \bigcap_{x \in X} B_{X^{* *}}(x,\|x-y\|) .
$$

It is a convex and weak ${ }^{*}$-compact subset of $X^{* *}$. Let $Z=\operatorname{span}(X,\{y\})$. There is a one-to-one correspondence between $D_{y}$ and $\mathrm{B}(X, Z)$ given by $\phi \leftrightarrow T_{\phi}(y)$. (If $d_{y} \in D_{y}$ define $T: Z \rightarrow X^{* *}$ by $T(a y+x)=a d_{y}+x$. See also Lemma 2.2 in [14].) Note that $D_{a y}=a D_{y}$ for $a \in \mathbb{R}$.

In view of the previous two propositions the following corollary is obvious.

Corollary 2.3. Assume $X$ is a u-ideal in $Y$. Then it is a strict u-ideal if and only if $D_{y} \subset S_{X^{* *}}$ for all $y \in S_{Y}$.

In Proposition 2.1 we saw that it is enough to check strictness of a u-ideal one direction at a time. Next we characterize strict $\mathrm{u}$-ideals of codimension one.

TheOREM 2.4. Let $X$ be a closed subspace of a Banach space $Y$. Let $y \in Y \backslash X$ and $Z=\operatorname{span}(X,\{y\})$. Assume that $X$ is a $u$-ideal in $Z$. The following statements are equivalent.

(a) $X$ is a strict $u$-ideal in $Z$.

(b) For every $z \in S_{Z}$ we have $\inf _{x \in S_{X}}\|z-2 x\|=1$.

(c) For every $z \in S_{Z}$ and $\varepsilon>0$ there exists $x \in S_{X}$ such that

$$
B_{X}(0,1-\varepsilon) \cap B_{X}(2 x,\|z-2 x\|)=\emptyset .
$$

Proof. (a) $\Rightarrow(\mathrm{b})$. Let $\phi \in \mathrm{B}(X, Z)$ be unconditional and strict and let $z \in S_{Z}$. Then $\left\|T_{\phi}(z)\right\|=1$ and by Lemma 2.2 in [9] there exists a net $\left(x_{\alpha}\right)$ in $X$ such that $\omega^{*}-\lim x_{\alpha}=T_{\phi}(z)$ and $\lim \sup _{\alpha}\left\|z-2 x_{\alpha}\right\| \leq 1$. Then

$$
2\left\|T_{\phi}(z)\right\| \leq 2 \liminf _{\alpha}\left\|x_{\alpha}\right\| \leq \limsup _{\alpha}\left\|2 x_{\alpha}\right\| \leq \limsup _{\alpha}\left\|z-2 x_{\alpha}\right\|+\|z\| \leq 2
$$

so we may assume that $x_{\alpha} \in S_{X}$ for all $\alpha$.

For all $x \in S_{X}$ we have $\|z-2 x\| \geq 2\|x\|-\|z\|=1$ but for $\varepsilon>0$ there is an $x_{\alpha}$ such that $\left\|z-2 x_{\alpha}\right\|<1+\varepsilon$. 
(b) $\Rightarrow$ (c). Let $z \in S_{Z}$ and $\varepsilon>0$. Choosing $x \in S_{X}$ with $\|z-2 x\|<1+\varepsilon$ we get $B_{X}(0,1-\varepsilon) \cap B_{X}(2 x,\|z-2 x\|)=\emptyset$.

(c) $\Rightarrow$ (a). We use Corollary 2.3. For all $z \in Z$ we have $D_{z} \subseteq B_{X^{* *}}(0,\|z\|)$ by definition. Let $z \in S_{Z}$. By (c) and the principle of local reflexivity we must have $B_{X^{* *}}(0,1-\varepsilon) \cap B_{X^{* *}}(2 x,\|z-2 x\|)=\emptyset$ and hence $D_{z} \cap B_{X^{* *}}(0,1-\varepsilon)=\emptyset$ for all $\varepsilon>0$.

Proposition 2.5. If $X$ is a (non-trivial) strict $u$-ideal in $Y$ and $P$ : $Y \rightarrow X$ is a projection then $\|P\| \geq 2$.

Proof. Assume that $P: Y \rightarrow X$ is a projection with norm $\|P\|=\lambda$. Let $y \in S_{Y} \cap \operatorname{ker} P$, let $\varepsilon>0$ and choose $x \in S_{X}$ such that

$$
B_{X}(0,1-\varepsilon) \cap B_{X}(2 x,\|y-2 x\|)=\emptyset
$$

using Theorem 2.4. We then get $\|2 x\|=\|P(y-2 x)\| \leq \lambda\|y-2 x\|$ so that $(2-2 / \lambda) x \in B_{X}(2 x,\|y-2 x\|)$. Then $(2-2 / \lambda) x \notin B_{X}(0,1-\varepsilon)$ and since $\varepsilon$ is arbitrary we get $2-2 / \lambda \geq 1$ or $\lambda \geq 2$.

Since dual spaces are 1-complemented in their biduals they can never be strict $\mathrm{u}$-ideals in their biduals. In fact, they cannot be a strict $\mathrm{u}$-ideal in any superspace.

Corollary 2.6. Assume that $X$ is a (non-trivial) u-ideal in $Y$. If $X$ is $\lambda$-complemented in its bidual with $\lambda<2$ then $X$ is not a strict $u$-ideal in $Y$.

Proof. Let $P: X^{* *} \rightarrow X$ be a projection with norm $\|P\|=\lambda$. Let $y \in Y \backslash X$ and $Z=\operatorname{span}(X,\{y\})$. Let $x^{* *} \in D_{y}$. Note that $D_{y}$ is non-empty since $\mathrm{B}(X, Y)$ is. Then for $x \in X$,

$$
\left\|P\left(x^{* *}\right)-x\right\| \leq \lambda\left\|x^{* *}-x\right\| \leq \lambda\|x-y\| .
$$

Hence $X$ is $\lambda$-complemented in $Z$ by the projection $Q: Z \rightarrow X$ defined by $Q(y)=P\left(x^{* *}\right)$ and $Q(x)=x$. From Propositions 2.5 and 2.1 we conclude that $X$ cannot be a strict u-ideal in $Y$.

Harmand and Lima [11, Theorem 3.5] showed that if $X$ is an M-ideal in its bidual then $X$ contains almost isometric copies of $c_{0}$ (i.e. $X$ has a subspace isomorphic to $c_{0}$ ). Next we generalize this to strict u-ideals. Note that the discussion regarding isometric copies of $c_{0}$ in [12, p. 79] also applies to strict $\mathrm{u}$-ideals.

Theorem 2.7. If $X$ is a (non-trivial) strict $u$-ideal in $Y$, then $X$ contains a copy of $c_{0}$.

Proof. If $X$ does not contain a copy of $c_{0}$ then $X$ is a u-summand in $Y$ by Theorem 3.5 in [9]. Using Proposition 2.5 gives us a contradiction.

The following is proved for separable Banach spaces and Banach spaces not containing $\ell_{1}$ in Proposition 5.2 in [9]. For every $X$ the natural embedding 
$k_{X^{*}}: X^{*} \rightarrow X^{* * *}$ is an element of $\mathrm{HB}\left(X, X^{* *}\right)$. We let $\pi: X^{* * *} \rightarrow X^{* * *}$ denote the associated ideal projection with $\operatorname{ker} \pi=X^{\perp}$.

Theorem 2.8. $X$ is a strict $u$-ideal in $X^{* *}$ if and only if $\|I-2 \pi\|=1$.

Proof. Assume that $X$ is a strict u-ideal in its bidual. Let $x^{* *} \in X^{* *} \backslash X$. We have $X \cap \bigcap_{x \in X} B_{X^{* *}}\left(x,\left\|x-x^{* *}\right\|\right)=\emptyset$ since any element in the intersection would define a norm one projection from $\operatorname{span}\left(X,\left\{x^{* *}\right\}\right)$ onto $X$, contradicting Proposition 2.5 (and Proposition 2.1).

By Lemma 2.4 in [10] we get $\bigcap_{x \in X} B_{X^{* *}}\left(x,\left\|x-x^{* *}\right\|\right)=\left\{x^{* *}\right\}$ and so the only element in $\mathrm{B}\left(X, X^{* *}\right)$ is $k_{X^{*}}$.

The other direction is trivial as $X^{*}$ is norming for $X^{* *}$.

REMARK 2.1. The above proof shows that if $X$ is a strict $\mathrm{u}$-ideal in its bidual then $\mathrm{HB}\left(X, X^{* *}\right)$ has only one element, i.e. the only extension operator is the trivial one $k_{X^{*}}$. In particular, the set $D_{x^{* *}}=\left\{x^{* *}\right\}$ is a singleton for every $x^{* *} \in X^{* *}$ (see (2.1), page 277 ).

The following theorem was inspired by Theorem 5.5 in [9]. The main improvement is that we remove the assumption that the space does not contain $\ell_{1}$.

TheOrem 2.9. Let $X$ be a Banach space. The following statements are equivalent.

(a) $X$ is a strict $u$-ideal in its bidual.

(b) Every subspace $Y$ of $X$ is a strict u-ideal in its bidual.

(c) For every subspace $Y$ of $X$ and $y^{* *} \in S_{Y^{* *}}$,

$$
\inf _{y \in S_{Y}}\left\|y^{* *}-2 y\right\|=1 \text {. }
$$

(d) Every separable subspace $Y$ of $X$ is a strict u-ideal in its bidual.

(e) For every separable subspace $Y$ of $X$ and $y^{* *} \in S_{Y^{* *}}$,

$$
\inf _{y \in S_{Y}}\left\|y^{* *}-2 y\right\|=1 .
$$

Proof. (b) $\Rightarrow(\mathrm{d})$ and $(\mathrm{c}) \Rightarrow(\mathrm{e})$ are trivial. (b) $\Rightarrow(\mathrm{c})$ and $(\mathrm{d}) \Rightarrow(\mathrm{e})$ follow from Theorem 2.4.

(a) $\Rightarrow$ (b). We use Theorem 2.8. Let $Y$ be a closed subspace of $X$ with natural embedding $i_{Y}: Y \rightarrow X$. By assumption $\left\|I-2 \pi_{X}\right\|=1$ where $\pi_{X}=k_{X^{*}} k_{X}^{*}$. We need to show that $\left\|I-2 \pi_{Y}\right\|=1$ where $\pi_{Y}=k_{Y^{*}} k_{Y}^{*}$. It is easy to check $i_{Y}^{* *} k_{Y}=k_{X} i_{Y}$ and $i_{Y}^{* * *} k_{X^{*}}=k_{Y^{*}} i_{Y}^{*}$ so that $i_{Y}^{* * *} \pi_{X}=\pi_{Y} i_{Y}^{* * *}$. We get

$$
1 \geq\left\|i_{Y}^{* * *}\left(I-2 \pi_{X}\right)\right\|=\left\|\left(I-2 \pi_{Y}\right) i_{Y}^{* * *}\right\| .
$$

Since $i_{Y}^{* *}: Y^{* *} \rightarrow X^{* *}$ is isometric, $i_{Y}^{* * *}$ is onto $Y^{* * *}$ and hence $\left\|I-2 \pi_{Y}\right\|=1$. $(\mathrm{e}) \Leftrightarrow(\mathrm{d})$ is proved in Theorem $5.5 \mathrm{in}[9]$. 
Finally, (d) $\Leftrightarrow$ (a) follows from Proposition 2.3 in [9] which characterizes strict u-ideals using sequences. Hence strict u-ideals are separably determined.

A quick look at Theorem 2.7 gives the following corollary.

Corollary 2.10. Assume that $X$ is non-reflexive. If $X$ is a strict u-ideal in its bidual then every non-reflexive subspace of $X$ contains a copy of $c_{0}$.

Remark 2.2. From Theorem 5.1 in [9] we know that a Banach space is not a strict $u$-ideal in its bidual if it contains $\ell_{1}$. The above corollary gives an alternative proof of this fact.

From Proposition 2.5 we know that $\ell_{\infty}$ is not a strict $\mathrm{u}$-ideal in its bidual. The next theorem shows that it is not even a u-ideal. We will also look at some consequences below.

\section{THEOREM 2.11. $\ell_{\infty}$ is not a u-ideal in its bidual.}

Before giving the proof of this theorem we need to introduce some more notation.

It is well-known that $\ell_{\infty}$ is isometrically isomorphic to $C(\beta \mathbb{N})$ where $\beta \mathbb{N}$ is the Stone-Cech compactification of the natural numbers (see e.g. Corollary 15.2 in [6]). The Riesz representation theorem identifies the dual with the measures on $\beta \mathbb{N}$. The state space of $C(\beta \mathbb{N})$ is the set

$$
S=\left\{x^{*} \in \ell_{\infty}^{*}:\left\|x^{*}\right\|=x^{*}(1)=1\right\},
$$

which is a weak*-closed subset of the dual unit ball. $S$ can be identified with the probability measures on $\beta \mathbb{N}$; the set of extreme points of $S$, ext $S$, is homeomorphic to $\beta \mathbb{N}$; and $S$ is a Bauer simplex (see e.g. [2, Corollary II.4.2]). $C(\beta \mathbb{N})$ is isometrically isomorphic to $A(S)$, the continuous affine functions on $S$ (see e.g. [2, Theorem II.1.8]). Thus for $f \in A(S)$ and $s \in S$ there is a unique probability measure $\mu$ on ext $S$ such that $f(s)=\int_{\text {ext } S} f d \mu$. We will write $s=r(\mu)$ where $r$ is the resultant (or barycenter) function. It is well-known that $S$ is a simplex (see e.g. [18, p. 53]) so $\mu$ is unique, i.e. $r$ is 1-1 ([18, Proposition 11.1]).

We say that a measure $\mu$ on $\beta \mathbb{N}$ is discrete if there is a countable set $\left\{z_{j}\right\}_{j=1}^{\infty} \subset \beta \mathbb{N}$ and numbers $\left\{a_{j}\right\}_{j=1}^{\infty}$ such that $\mu=\sum_{j=1}^{\infty} a_{j} \delta_{z_{j}}$. On the other hand, $\mu$ is continuous if $\mu(\{z\})=0$ for all $z \in \beta \mathbb{N}$. Any measure $\mu$ can be written uniquely as $\mu=\mu_{d}+\mu_{c}$ where $\mu_{d}$ is discrete and $\mu_{c}$ is continuous by letting $E=\{z: \mu(\{z\}) \neq 0\}$ and defining $\mu_{d}(A)=\mu(A \cap E)$ and $\mu_{c}(A)=\mu(A \backslash E)$. Since $\mathbb{N}$ is countable we can write $\mu_{d}=\mu_{n d}+\mu_{b d}$ where $\mu_{n d}(A)=\mu_{d}(A \cap \mathbb{N})$ and $\mu_{b d}(A)=\mu_{d}(A \backslash \mathbb{N})$. 
We will define the following faces of $S$ :

$$
\begin{aligned}
& S_{1}=\left\{s \in S: s=r\left(\mu_{n d}\right), \text { a discrete measure on } \mathbb{N}\right\}, \\
& S_{2}=\left\{s \in S: s=r\left(\mu_{b d}\right), \text { a discrete measure on } \beta \mathbb{N}\right\}, \\
& S_{3}=\left\{s \in S: s=r\left(\mu_{c}\right), \text { a continuous measure on } \beta \mathbb{N}\right\} .
\end{aligned}
$$

We have $S=\operatorname{conv}\left(\bigcup_{i=1}^{3} S_{i}\right)$ and $S_{i} \cap S_{j}=\emptyset$ for $i \neq j$. We will also need the complementary face of $S_{i}$, namely $S_{i}^{\prime}=\operatorname{conv}\left(\bigcup_{i \neq j} S_{i}\right)$. (Here we have used that closed faces in a simplex are split; see [2, pp. 132-133, Proposition II.6.7 and Corollary II.6.8] and [4, p. 140, Theorem 8.3].) Also note that $S_{3} \neq \emptyset$ since we can pull back Lebesgue measure from $C[0,1]^{*}$.

Proof of Theorem 2.11. We identify $\ell_{\infty}$ with $A(S)$ and $A(S)^{* *}$ with the bounded affine functions on $S, A_{b}(S)$. (This is "easy to check" [4, p. 43].) Each $s \in S$ can be written uniquely as $s=\alpha_{i} s_{i}+\left(1-\alpha_{i}\right) s_{i}^{\prime}$ where $\alpha_{i} \in[0,1]$, $s_{i} \in S_{i}$ and $s_{i}^{\prime} \in S_{i}^{\prime}$. Thus the functions $f_{i}(s)=2 \alpha_{i}-1$ are well-defined and $f_{i} \in A_{b}(S)$. We will use that $f_{i}=1$ on $S_{i}$ and $f_{i}=-1$ on $S_{i}^{\prime}$.

Assume for contradiction that $\ell_{\infty}$ is a u-ideal in its bidual. Define $H=$ $\operatorname{span}\left(f_{i}\right)_{i=1}^{3}$, a subspace of $\ell_{\infty}^{* *}$, and let $\varepsilon>0$.

By the local characterization of u-ideals (Proposition 3.6 in [9]), there is an operator $L: H \rightarrow \ell_{\infty}$ such that $\|L\| \leq 1+\varepsilon,\|h-2 L(h)\| \leq(1+\varepsilon)\|h\|$ for all $h \in H$ and $L(x)=x$ for all $x \in H \cap \ell_{\infty}$. Since $L(1)=1$ we get $\sum_{i=1}^{3} L\left(f_{i}\right)=-1$.

Using $\left\|f_{i}-2 L\left(f_{i}\right)\right\| \leq(1+\varepsilon)\left\|f_{i}\right\| \leq 1+\varepsilon$ we see that on $S,-(1+\varepsilon) \leq$ $-f_{i}+2 L\left(f_{i}\right) \leq 1+\varepsilon$ or $f_{i}-1-\varepsilon \leq 2 L\left(f_{i}\right) \leq f_{i}+1+\varepsilon$. So on $S_{i}$ we have $-\varepsilon / 2 \leq L\left(f_{i}\right) \leq 1+\varepsilon / 2$.

By density of $\mathbb{N}$ in its compactification $\beta \mathbb{N}$ we must have $L\left(f_{1}\right) \geq-\varepsilon / 2$ on $\beta \mathbb{N}$ since $L\left(f_{1}\right) \geq-\varepsilon / 2$ on $\mathbb{N}$. Also, we have $L\left(f_{2}\right) \geq-\varepsilon / 2$ on $\beta \mathbb{N} \backslash \mathbb{N}$. Since $\mathbb{N}$ is countable the continuous measure $\mu$ corresponding to $s \in S_{3}$ has support on $\beta \mathbb{N} \backslash \mathbb{N}$ so

$$
L f_{i}(s)=\int_{\text {ext } S} L f_{i} d \mu=\int_{\beta \mathbb{N} \backslash \mathbb{N}} L f_{i} d \mu \geq-\varepsilon / 2
$$

for $i=1,2$. Thus on $S_{3}$ we have

$$
-\varepsilon / 2 \leq L\left(f_{3}\right)=-1-L\left(f_{1}\right)-L\left(f_{2}\right) \leq-1+\varepsilon,
$$

or $0 \leq-1+3 \varepsilon / 2$. Since $\varepsilon>0$ is arbitrary this is a contradiction.

REMARK 2.3. Since $\ell_{\infty}$ is injective, $\ell_{\infty}$ is never a strict $\mathrm{u}$-ideal in $Z=$ $\operatorname{span}\left\{\ell_{\infty}, f\right\}$ for $f \in \ell_{\infty}^{* *}$. In some cases it is a u-ideal, however.

In the notation above, set $f=1$ on $S_{1}$ and $f=-1$ on $S_{1}^{\prime}=\operatorname{conv}\left(S_{2} \cup S_{3}\right)$. Let $\varepsilon>0, x_{i} \in \ell_{\infty}$ and $r_{i}=\left\|f-x_{i}\right\|$ for $i=1,2,3$. Without loss of generality we may assume that $x_{i}=\sum_{k=1}^{m} a_{i, k} \chi_{A_{k}}$ where $A_{k}$ is a partition of $\mathbb{N}$ (use an 
$\varepsilon$-net on the set $\left(x_{i}(n)\right)_{n=1}^{\infty}$ if necessary). We may assume that $A_{1}, \ldots, A_{p}$ are finite sets and that $A_{p+1}, \ldots, A_{m}$ are infinite.

Define an element $x \in \ell_{\infty}$ by setting $x_{n}=2$ for $n \in \bigcup_{k=1}^{p} A_{k}$ and $x_{n}=0$ for $n \in \bigcup_{k=p+1}^{m} A_{k}$. Then $x \in \ell_{\infty} \cap \bigcap_{i=1}^{3} B_{Z}\left(f+x_{i}, r_{i}+\varepsilon\right)$ and by Theorem 1.3 in [15], $\ell_{\infty}$ is a u-ideal in $Z$.

As noted in Proposition 2.5, a non-reflexive dual space can never be a strict $\mathrm{u}$-ideal. Using that $\ell_{\infty}$ is not a $\mathrm{u}$-ideal in its bidual we can say even more.

Theorem 2.12. Let $X$ be a Banach space such that $X^{*}$ is a $u$-ideal in its bidual. Then $X^{*}$ is a u-summand.

Proof. If $X^{*}$ contains a copy of $c_{0}$ then it contains a copy of $\ell_{\infty}$ by Bessaga and Pełczyński [5]. By Partington [16] and Talagrand [19, Theorem 6] (and injectivity) it has $(1+\varepsilon)$-complemented copies of $\ell_{\infty}$ for every $\varepsilon>0$. The local characterization of u-ideals (Proposition 4.1 in [9]) would then imply that $\ell_{\infty}$ is a u-ideal in its bidual, which is impossible by Theorem 2.11. Hence $X^{*}$ is a u-ideal not containing $c_{0}$, so it is a u-summand by Theorem 3.5 in [9].

Remark 2.4. Assume $X$ is a strict u-ideal in its bidual. Then $\|I-2 \pi\|$ $=1$ and considering the adjoint projection $P=\pi^{*}$ on $X^{(4)}$ we have $\operatorname{ker} P=$ $(\operatorname{im} \pi)^{\perp}=\left(X^{*}\right)^{\perp}$. Since $\|I-2 P\|=\|I-2 \pi\|=1$ we conclude that $X^{*}$ is a $\mathrm{u}$-ideal in its bidual and by the above theorem even a u-summand.

We do not know whether $X$ a u-ideal in its bidual and $X^{*}$ a u-summand in its bidual implies that $X$ is a strict u-ideal.

3. Geometric properties. A slice of a bounded, closed, convex subset $C$ of $X$ is a subset $S\left(C, x^{*}, \alpha\right)$ of $C$ defined by

$$
S\left(C, x^{*}, \alpha\right)=\left\{x \in C: x^{*}(x)>\sup _{y \in C} x^{*}(y)-\alpha\right\},
$$

where $x^{*} \in X^{*} \backslash\{0\}$ and $\alpha>0$. If $X$ is a dual space we can speak of a weak ${ }^{*}$ slice when the defining functional is weak*-continuous. A bounded, closed, convex set $C$ is dentable if it has slices of arbitrarily small diameter. Recall that the diameter of a non-empty set $A$ is given by $\operatorname{diam}(A)=\sup \{\|x-y\|$ : $x, y \in A\}$. A point $x \in C$ is called a denting point in $C$ if there is a sequence of slices $S_{n}$ of $C$ with $x \in S_{n}$, for all $n$, such that $\operatorname{diam}\left(S_{n}\right) \rightarrow 0$. If $C$ is a subset of a dual space $X^{*}$ then $x^{*} \in C$ is a weak*-denting point in $C$ if there is a sequence of weak*-slices $S_{n}$ of $C$ with $x^{*} \in S_{n}$ for all $n$ such that $\operatorname{diam}\left(S_{n}\right) \rightarrow 0$. A point $x \in C$ is called a strongly exposed point in $C$ if there is an $x^{*} \in X^{*}$ such that $x^{*}(x)>x^{*}(y)$ for all $x \neq y \in C$ and $\operatorname{diam}\left(S\left(C, x^{*}, \alpha\right)\right) \rightarrow 0$ as $\alpha \rightarrow 0$. Weak* strongly exposed points are defined in the obvious way. 
By definition $\omega^{*}$-str.exp. $B_{X^{*}} \subset \omega^{*}$-dent. $B_{X^{*}}$. When $X$ is a strict u-ideal in its bidual we can say much more. The next proposition highlights that this is a really strong geometric property.

Proposition 3.1. Assume that $X$ is a strict u-ideal in its bidual. Then str.exp. $B_{X^{*}} \subset \omega^{*}$-dent. $B_{X^{*}}$.

Proof. Let $x^{*} \in$ str.exp. $B_{X^{*}}$ and let $x^{* *} \in S_{X^{* *}}$ be a strongly exposing functional for $x^{*}$. Let $\varepsilon>0$ and choose $\delta_{0}>0$ such that $\left\{u^{*} \in B_{X^{*}}\right.$ : $\left.x^{* *}\left(u^{*}\right)>1-\sqrt{\delta_{0}}\right\} \subset B_{X^{*}}\left(x^{*}, \varepsilon\right)$ and $1+\varepsilon \delta_{0}>2 \sqrt{\delta_{0}}(1+\varepsilon)$.

Let $\delta \in\left(0, \delta_{0}\right)$. Then $1+\varepsilon \delta>2 \sqrt{\delta}(1+\varepsilon)$, which is equivalent to $2(1-\delta) /(1+\varepsilon \delta)-2+\sqrt{\delta}>0$. Choose $\eta>0$ with $0<\eta<2(1-\delta) /(1+\varepsilon \delta)$ $-2+\sqrt{\delta}$ and $\left\{u^{*} \in B_{X^{*}}: x^{* *}\left(u^{*}\right)>1-\eta\right\} \subset B_{X^{*}}\left(x^{*}, \varepsilon \delta /(1+\varepsilon \delta)\right)$.

Since $X$ is a strict u-ideal we have $1=\inf _{x \in S_{X}}\left\|x^{* *}-2 x\right\|$. Choose $x \in S_{X}$ such that $\left\|x^{* *}-2 x\right\|<1+\eta$. Choose $u^{*} \in B_{X^{*}}$ such that $u^{*}(x)=1$. Then

$$
1+\eta>\left\|x^{* *}-2 x\right\| \geq u^{*}\left(2 x-x^{* *}\right)=2-x^{* *}\left(u^{*}\right) .
$$

Thus $x^{* *}\left(u^{*}\right)>1-\eta$. It follows that $\left\|u^{*}-x^{*}\right\|<\varepsilon \delta /(1+\varepsilon \delta)$.

Let $u=x / x^{*}(x)$. Then $x^{*}(x) \geq u^{*}(x)-\left\|x^{*}-u^{*}\right\|>1 /(1+\varepsilon \delta)$ so $\|u\|=1 / x^{*}(x) \leq 1+\varepsilon \delta$. If $z^{*} \in B_{X^{*}}$ and $z^{*}(u)>1-\delta$, then $z^{*}(x)=$ $z^{*}(u) x^{*}(x)>(1-\delta) x^{*}(x)$. Hence

$$
1+\eta>\left\|x^{* *}-2 x\right\| \geq z^{*}\left(2 x-x^{* *}\right) \geq 2(1-\delta) x^{*}(x)-x^{* *}\left(z^{*}\right),
$$

and $x^{* *}\left(z^{*}\right)>2(1-\delta) x^{*}(x)-1-\eta \geq 2(1-\delta) /(1+\varepsilon \delta)-1-\eta$. But then $x^{* *}\left(z^{*}\right)>1-\sqrt{\delta}$, from which it follows that $\left\|z^{*}-x^{*}\right\|<\varepsilon$. Thus $x^{*}$ is contained in weak ${ }^{*}$-slices of arbitrarily small diameter, i.e. $x^{*}$ is weak ${ }^{*}$-denting.

Next we use the weak*-denting points in the unit ball to characterize when a $u$-ideal is a strict $u$-ideal. For Banach spaces not containing $\ell_{1}$ the equivalence of (a) and (d) was proved in Theorem 7.4 in [9].

Proposition 3.2. Let $X$ be a Banach space. Assume that $X$ is a u-ideal in its bidual. Then the following are equivalent.

(a) $X$ is a strict $u$-ideal in its bidual.

(b) $B_{X^{*}}=\overline{\operatorname{conv}}\left(\omega^{*}\right.$-str.exp. $\left.B_{X^{*}}\right)$.

(c) $B_{X^{*}}=\overline{\operatorname{conv}}\left(\omega^{*}\right.$-dent. $\left.B_{X^{*}}\right)$.

(d) $X^{*}$ contains no proper norming subspaces.

(e) $T_{\phi}=I_{X^{* *}}$ where $\phi \in \mathrm{H}\left(X, X^{* *}\right)$ is the unconditional extension operator.

Proof. (a) $\Rightarrow$ (b) follows from Theorem 2.8 and Proposition 4.1 in [13].

$(\mathrm{b}) \Rightarrow(\mathrm{c})$ is trivial.

(c) $\Rightarrow$ (a). The weak*-denting points have unique norm-preserving extension so $\mathrm{B}\left(X, X^{* *}\right)=\left\{k_{X^{*}}\right\}$. $X$ is a strict u-ideal by Theorem 2.8.

(a) $\Rightarrow(d)$. Follows from Theorem 2.8 and Proposition 2.7 in [9]. 
$(\mathrm{d}) \Rightarrow(\mathrm{e})$. By Proposition 2.5 in [10], $X$ has the unique extension property and by definition the only contractive operator $T: X^{* *} \rightarrow X^{* *}$ with $\left.T\right|_{X}=$ $I_{X}$ is $T=I_{X^{* *}}$.

(e) $\Rightarrow(\mathrm{a}) . \quad X$ is a strict $\mathrm{u}$-ideal by Theorem 2.8 .

REMARK 3.1. The dual of a Banach space $X$ has the Radon-Nikodým property if and only if every separable subspace of $X$ has separable dual (see e.g. [7, Corollary VII.2.8]). This is the case if $X$ is a strict u-ideal in its bidual (see e.g. Proposition 4.1 in [13] or Proposition 2.8 in [9]).

On the other hand, if $X^{*}$ has the Radon-Nikodým property then $B_{X^{*}}=$ $\overline{\mathrm{conv}} w^{*}\left(\omega^{*}\right.$-str.exp. $\left.B_{X^{*}}\right)$ [17, Theorem 5.12]. We do not know if this is enough to ensure that a $\mathrm{u}$-ideal is strict.

It is also an open problem whether a u-ideal is strict if the space does not contain $\ell_{1}$ (see Question 5 in [9]).

Acknowledgements. We would like to thank the referee for helpful comments that helped improve the manuscript.

\section{References}

[1] T. A. Abrahamsen, V. Lima, and Å. Lima, Unconditional ideals of finite rank operators, Czechoslovak Math. J. 58 (2008), 1257-1278.

[2] E. M. Alfsen, Compact Convex Sets and Boundary Integrals, Springer, Berlin, 1971.

[3] E. M. Alfsen and E. G. Effros, Structure in real Banach spaces. Parts I and II, Ann. of Math. 96 (1972), 98-173.

[4] L. Asimow and A. J. Ellis, Convexity Theory and its Applications in Functional Analysis, London Math. Soc. Monogr. 16, Academic Press, London, 1980.

[5] C. Bessaga and A. Pełczyński, On bases and unconditional convergence of series in Banach spaces, Studia Math. 17 (1958), 151-164.

[6] N. L. Carothers, A Short Course on Banach Space Theory, London Math. Soc. Student Texts 64, Cambridge Univ. Press, Cambridge, 2005.

[7] J. Diestel and J. J. Uhl, Vector Measures, Math. Surveys 15, Amer. Math. Soc., Providence, RI, 1977.

[8] H. Fakhoury, Sélections linéaires associées au théorème de Hahn-Banach, J. Funct. Anal. 11 (1972), 436-452.

[9] G. Godefroy, N. J. Kalton, and P. D. Saphar, Unconditional ideals in Banach spaces, Studia Math. 104 (1993), 13-59.

[10] G. Godefroy and P. D. Saphar, Duality in spaces of operators and smooth norms on Banach spaces, Illinois J. Math. 32 (1988), 672-695.

[11] P. Harmand and Å. Lima, Banach spaces which are $M$-ideals in their biduals, Trans. Amer. Math. Soc. 283 (1984), 253-264.

[12] P. Harmand, D. Werner, and W. Werner, M-Ideals in Banach Spaces and Banach Algebras, Lecture Notes in Math. 1547, Springer, Berlin, 1993.

[13] A. Lima, Property $\left(w M^{*}\right)$ and the unconditional metric compact approximation property, Studia Math. 113 (1995), 249-263.

[14] A. Lima and E. Oja, Ideals of finite rank operators, intersection properties of balls, and the approximation property, ibid. 133 (1999), 175-186. 
[15] V. Lima and A. Lima, A three ball intersection property for u-ideals, J. Funct. Anal. 251 (2007), 220-232.

[16] J. R. Partington, Subspaces of certain Banach sequence spaces, Bull. London Math. Soc. 13 (1981), 163-166.

[17] R. R. Phelps, Convex Functions, Monotone Operators and Differentiability, Lecture Notes in Math. 1364, Springer, Berlin, 1989.

[18] - Lectures on Choquet's Theorem, 2nd ed., Lecture Notes in Math. 1757, Springer, Berlin, 2001.

[19] M. Talagrand, Sur les espaces de Banach contenant $l^{1}(\tau)$, Israel J. Math. 40 (1981), $324-330$.

Aalesund University College

Service Box 17

N-6025 Ålesund, Norway

E-mail: Vegard.Lima@gmail.com
Department of Mathematics University of Agder Serviceboks 422 4604 Kristiansand, Norway E-mail: Asvald.Lima@uia.no

Received January 23, 2009

Revised version August 17, 2009 